\title{
Aging-related magnification of genetic effects on cognitive and brain integrity
}

\author{
Goran Papenberg ${ }^{1}$, Ulman Lindenberger ${ }^{2,3}$, and Lars Bäckman ${ }^{1}$ \\ ${ }^{1}$ Aging Research Center, Karolinska Institutet and Stockholm University, Stockholm, Sweden \\ ${ }^{2}$ Center for Lifespan Psychology, Max Planck Institute for Human Development, Berlin, Germany \\ ${ }^{3}$ Max Planck University College London Centre for Computational Psychiatry and Ageing Research, London, UK
}

Heritability studies document substantial genetic influences on cognitive performance and decline in old age. Increasing evidence shows that effects of genetic variations on cognition, brain structure, and brain function become stronger as people age. Disproportionate impairments are typically observed for older individuals carrying disadvantageous genotypes of different candidate genes. These data support the resource-modulation hypothesis, which states that genetic effects are magnified in persons with constrained neural resources, such as older adults. However, given that findings are not unequivocal, we discuss the need to address several factors that may resolve inconsistencies in the extant literature (genegene and gene-environment interactions, study populations, gene-environment correlations, and epigenetic mechanisms).

Inter-individual differences in cognitive and brain aging Human aging is characterized by large and increased interindividual differences in different aspects of cognitive performance, brain structure, and brain function [1-3]. Whereas some older individuals may have cognitive abilities that match those of younger individuals, older persons of the same age may show rapid decline in cognitive and brain integrity $[3,4]$. Conceivably, multiple factors contribute to individual differences at neural and behavioral levels, including genetic predispositions and lifestyle factors. In recent years evidence has accumulated that the effects of common genetic variations may increase in aging, contributing to inter-individual neural and cognitive differences among older adults.

\section{Heritability estimates of cognitive and brain measures in old age}

Heritability studies demonstrate increased genetic influences on different types of cognition in aging [5-7], and also regarding the rate of cognitive decline [8]. Meta-analytic evidence suggests increased heritability from early to late adulthood, especially for episodic memory (see Glossary), but also for working memory and spatial ability [6]. In addition, it has been shown that one-third of individual

Corresponding author: Papenberg, G. (goran.papenberg@ki.se).

Keywords: aging; cognition; genetics; brain structure; brain function.

1364-6613/

(c) 2015 Elsevier Ltd. All rights reserved. http://dx.doi.org/10.1016/j.tics.2015.06.008 differences in global cognitive changes from 65 to 96 years of age are attributable to genetic factors [8]. Concerning brain measures, available aging data are sparse, although studies generally suggest decreasing heritability estimates across the adult lifespan, followed by increases in late adulthood for global brain volumes [9]. Genetic estimates of ventricular volume, an indirect measure of brain volume, have also revealed increasing heritability in old age [10].

\section{Glossary}

Allele: different forms of a gene are termed alleles.

Candidate gene: a gene whose function has been implicated in a particular phenotype of interest, such as brain and cognitive function.

Cognitive dedifferentiation: aging-related increase in correlations between different cognitive domains. A common mechanism or an ensemble of common mechanisms may lead to decline in different cognitive processes, and consequently to a higher degree of dedifferentiation across domains of functioning.

Diffusion tensor imaging (DTI): neuroimaging technique sensitive to the diffusion of water molecules within the architecture of the tissue. It allows the assessment of degree of anisotropy and structural orientation that characterize diffusion tensor imaging. Fractional anisotropy (FA) indicates directionality of diffusion, and mean diffusivity (MD) indicates diffusion, independent of directionality. Higher white-matter integrity is associated with higher FA and lower MD.

Epigenetics: study of how external or environmental factors influence gene expression, for instance through changes in DNA methylation.

Episodic memory: ability to recall specific past events that are localized in time and space.

Executive functioning: complex cognitive process, including different subprocesses such as inhibition of a response, updating of working-memory representations, and ability to flexibly shift between different tasks or cognitive operations.

Functional magnetic resonance imaging (fMRI): neuroimaging method that allows measurement of neural activity by detecting associated changes in blood flow and changes in deoxyhemoglobin levels, which are reflected in the blood-oxygen-level-dependent (BOLD) signal.

Genome-wide association study (GWAS): examination of multiple common genetic variants across the entire genome for their association with a particular trait.

Genotype: the identity of the two alleles at a specific genetic locus.

Global cognitive ability: broad intellectual ability that mainly represents reasoning, but also other cognitive domains, including memory, processing speed, and verbal comprehension.

Heterozygote: a carrier of two different alleles at a specific genetic locus Homozygote: a carrier of two identical alleles at a specific genetic locus. Mild cognitive impairment (MCl): individuals with $\mathrm{MCl}$ are characterized by more severe cognitive decline than would be expected in normal aging and are at an increased risk of developing dementia.

Prodromal dementia/Alzheimer disease (AD): stage of dementia or AD before a clinical diagnosis may be rendered that is characterized by mild symptoms typical for the disease.

Single-nucleotide polymorphism (SNP): a variation at a single position in a deoxyribonucleic acid (DNA) sequence.

Working memory: ability to consciously maintain and manipulate information in mind. 
Although effects of common genetic variations are small $(<1 \%$ of explained variance), overall they still account for a considerable amount of phenotypic variance. Heritability estimates based on single-nucleotide polymorphisms (SNPs) for cognitive measures range between $31 \%$ and $51 \%$, indicating substantial heritability for behavioral measures $[11,12]$.

SNP-based heritability is typically lower than heritability estimates based on twin studies [6], the latter reflecting both general effects of specific genes and gene-gene interactions. By contrast, estimations based on SNPs alone do not capture gene-gene interactions, likely resulting in this discrepancy. Interestingly, heritability for cognition seems to decrease once individuals reach dementia or terminal decline. Genetic contributions to different forms of memory are smaller in samples of individuals with Alzheimer's disease $(\mathrm{AD})$ and their unaffected family members than for unaffected family members alone [13], suggesting that genes account less for individual differences in $\mathrm{AD}$ patients.

\section{The resource-modulation hypothesis}

The resource-modulation hypothesis, introduced by Lindenberger and colleagues, posits that losses of anatomical and neurochemical brain resources in normal aging modulate the effects of common genetic variations on cognitive functioning [14]. This notion is based on the assumption that the function relating brain resources to cognition is non-linear, and that genetic differences therefore exert increasingly larger effects on performance as resources recede from high to medium levels (Figure 1). Given that neural measures of brain structure and function may be closer to the molecular effects of a gene than cognitive measures, they are expected to be more sensitive to genetic effects [15]. Thus, older adults may benefit more from beneficial genetic predispositions relative to younger adults, and thereby be able to maintain brain and cognitive functioning in senescence.

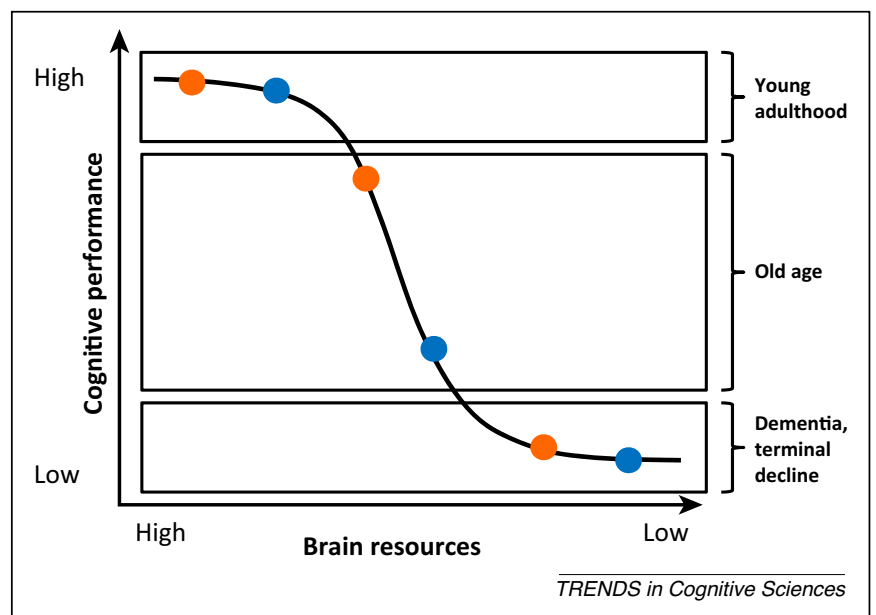

Figure 1. The resource-modulation hypothesis assumes that the function relating brain resources to cognition is non-linear and predicts magnified genetic effects on cognitive performance in old age. In healthy aging, associated with decline in anatomical and chemical brain resources, constant amounts of genetic variation translate into increasingly large performance differences. With resources further depleted, genetic effects are expected to diminish. The colored circles represent two hypothetical individuals with different genetic predispositions as they move from early adulthood through old age to dementia or terminal decline. Adapted from [14] with permission from Frontiers Research Foundation.

\section{Support for aging-related magnification of genetic effects on brain and behavior}

Increasing evidence from behavioral, structural, and functional imaging studies supports the resource-modulation hypothesis. The bulk of studies suggest that effects of genetic variations are either small or not detectable in younger adults, but become magnified in old age, with older carriers of disadvantageous genotypes declining disproportionately with respect to brain and cognition. We next review these effects with examples involving different candidate genes.

\section{Apolipoprotein E (APOE) polymorphism}

APOE is a lipoprotein involved in many steps of lipid homeostasis and injury repair in the brain [16]. The e4 allele of $A P O E$ is a strong risk factor for $\mathrm{AD}[17,18]$, and is associated with accelerated cognitive decline in normal aging $[19,20]$. A meta-analysis showed that e4 carriers have lower performance on several cognitive measures [21]. Crucially, $A P O E$-related effects were more pronounced in older than younger individuals with respect to global cognitive ability and episodic memory. In line with this pattern, longitudinal studies have documented interactions between age and $A P O E$, with increasing negative effects of e 4 in persons older than 50 years on learning and episodic memory (Figure 2) [22]. In another study, e4 carriers showed exacerbated decline in verbal memory and reasoning between 79 and 87 years of age [23]. So far, most genome-wide association studies (GWAS) with healthy adults have not used cognitive decline as the outcome or stratified the data across age groups. However, two GWAS demonstrated effects of APOE on rate of cognitive decline $[24,25]$, thus supporting the magnification view.

Stronger effects of $A P O E$ in old age are also seen at the neural level. An fMRI study reported an interaction between age and $A P O E$ status during encoding of episodic memories, with e4 carriers showing decreased activation in multiple brain regions including the hippocampus, an area crucial for successful episodic memory [26]. Notably, these findings were independent of individual differences in

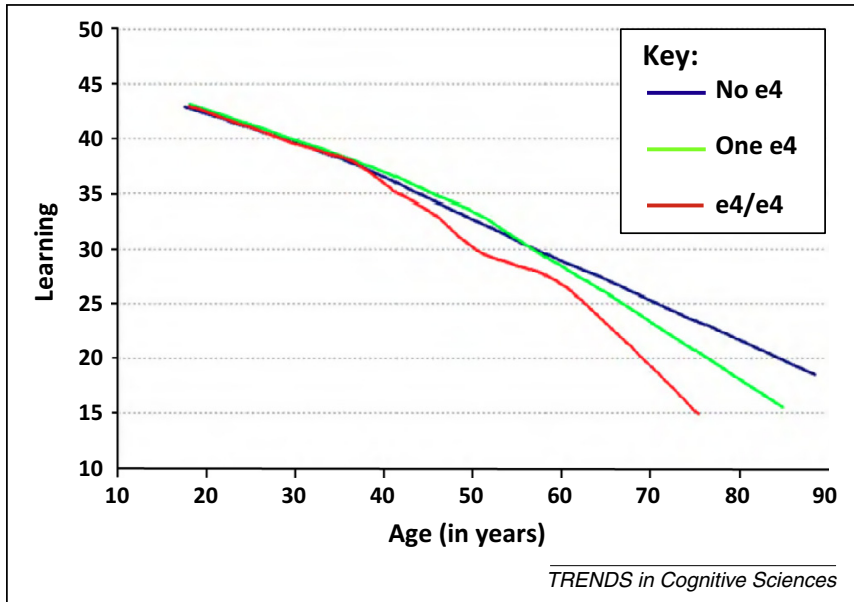

Figure 2. Effects of the apolipoprotein $\mathrm{E}(A P O E)$ polymorphism on learning, with increased negative dose-response effects of the e4 allele across adult age Learning reflects the number of correctly recalled words in the Rey Auditory Verbal Learning Test. Adapted from [22] with permission from Elsevier. 
gray-matter volumes. However, data from another lifespan fMRI study suggested an opposite pattern for hippocampal activation in older adults [27]. In this study there was decreased hippocampal activity during encoding and retrieval of neutral pictures with increasing age, and these decreases were weaker for e4 carriers than for non-carriers. These two studies provide evidence in line with the resource-modulation hypothesis, although they document opposing genetic effects on neural functioning in old age. One source of variation that may account for this discrepancy between studies is task difficulty. Older adults at higher risk for cognitive decline typically show more brain activity during relatively simple tasks than individuals at lower risk. However, during more difficult tasks, the pattern may be reversed [28]. In line with this notion, participants were instructed to remember images in the study where older e4 carriers had lower brain activity at encoding [26]. This task is clearly more cognitively challenging than judging the contents of images during study, a task for which greater brain activity in older e4 carriers was observed [27]. Concerning structural brain-imaging markers, longitudinal studies demonstrate more hippocampal atrophy for e 4 carriers $[29,30]$ that may contribute to the effects of $A P O E$ on functional brain activity. Consistent with the assumption that genetic effects diminish once individuals reach very low cognitive-performance levels (Figure 1), evidence indicates that $A P O E$ does not affect progression rate in clinical $\mathrm{AD}$ [31], or even the rate of decline from preclinical to clinical dementia [32].

Brain-derived neurotrophic factor (BDNF) polymorphism BDNF promotes synaptic plasticity and is crucial for hippocampus-dependent learning and memory [33]. Variation in the $B D N F$ gene is associated with individual differences in secretion of this protein, which is greater in Val homozygotes than in Met carriers [34]. Meta-analytic evidence confirms negative, albeit small, effects of the BDNF Met allele on human episodic memory [35]. Importantly, age-comparative studies have reported magnified effects of $B D N F$ in old age, with older Val homozygotes showing better episodic memory compared with older Met carriers [36]. In addition, in line with the resource-modulation hypothesis, longitudinal data from a sample of older adults aged 70 to 103 years demonstrate exacerbated decline in perceptual speed across 13 years for Met carriers [37], an effect that remained after excluding prodromal dementia cases (Figure 3A). Similarly, pilots carrying the Met allele (aged 40-69 years) declined disproportionately across 2 years in flight-simulator performance, presumably reflecting executive functioning [38]. At the neural level, $B D N F$ Met carriers exhibited lower hippocampal activity during encoding and retrieval of episodic memories [35]. A study with persons across the entire adult lifespan documented larger decreases in hippocampal activity with advancing age for Met carriers than for Val homozygotes during both encoding and retrieval of episodic memories (Figure 3B) [39], which was independent of inter-individual differences in hippocampal volume. Age magnification of the effects of $B D N F$ has also been reported for other measures of brain integrity, emphasizing the role of BDNF in modulating myelin expression [40] and survival of neurons in the adult brain [41]. Specifically, Met carriers had lower hippocampal volumes than Val homozygotes after age 65, whereas no such differences were apparent at younger ages (Figure 3C) [38]. Crucially, age was unrelated to hippocampal volume in Val homozygotes, supporting the idea that brain maintenance in old age may partly reflect genetic factors [4]. Another study with individuals in the prodromal phase of $\mathrm{AD}$ reported that the Met allele was associated with increased memory decline across 3 years, paralleled by more hippocampal atrophy [42]. Similarly, age-related decline in white-matter microstructure, as measured with diffusion tensor imaging, was found for Met carriers, although no such decline was evident for Val homozygotes (Figure 3D) [43].

Taken together, accumulating evidence suggests increased effects of $B D N F$ on brain and cognition in aging, with greater decline in performance for older Met carriers.

\section{Catechol-O-methyltransferase (COMT) polymorphism}

COMT is involved in extracellular degradation of dopamine (DA) in prefrontal cortex (PFC) [44,45]. DA concentrations modulate neuronal signal-to-noise ratio in the $\mathrm{PFC}$ that is crucial for efficient cognitive processing [46]. COMT Val homozygotes have 3-4-fold higher DA-degrading activity than Met homozygotes [47], resulting in lower prefrontal DA availability and presumably less efficient cognitive processing. Cross-sectional studies demonstrate faster response times [48] and higher accuracy during working memory [49] for older Met homozygotes than for Val carriers, whereas no difference was found between genotype groups among younger individuals. Another study found that older COMT Val homozygotes had a strong correlation between episodic and working memory, indicating cognitive dedifferentiation. By contrast, older Met carriers and younger COMT genotype groups had identical and considerably weaker correlations between the two types of memory [50]. Most importantly, longitudinal data reveal less decline of executive functions over a 5 -year interval [51], and less decline of episodic memory across 15 years [52], for older Met carriers than for Val homozygotes. Results from an fMRI study further suggest that older Val homozygotes are characterized by less efficient processing during a working memory task with low demands, as indicated by increased communication between distal brain regions, compared with Met homozygotes, potentially reflecting a compensatory response [53]. Again, these group differences were not present in younger adults. Relatedly, a structural imaging study in a population-based sample found that COMTVal status was associated with reduced white-matter integrity in several prefrontal white-matter tracts in old age, although there were no reliable associations between COMT and whitematter integrity in younger age groups [54].

\section{Kidney and brain expressed protein (KIBRA) polymorphism}

Genetic variation in the KIBRA gene has been associated with episodic memory, with T-allele carriers exhibiting better performance than C-allele homozygotes [55]. In the human brain, KIBRA is mainly expressed in hippocampus and interacts with proteins involved in long-term potentiation, a cellular mechanism necessary for successful memory 

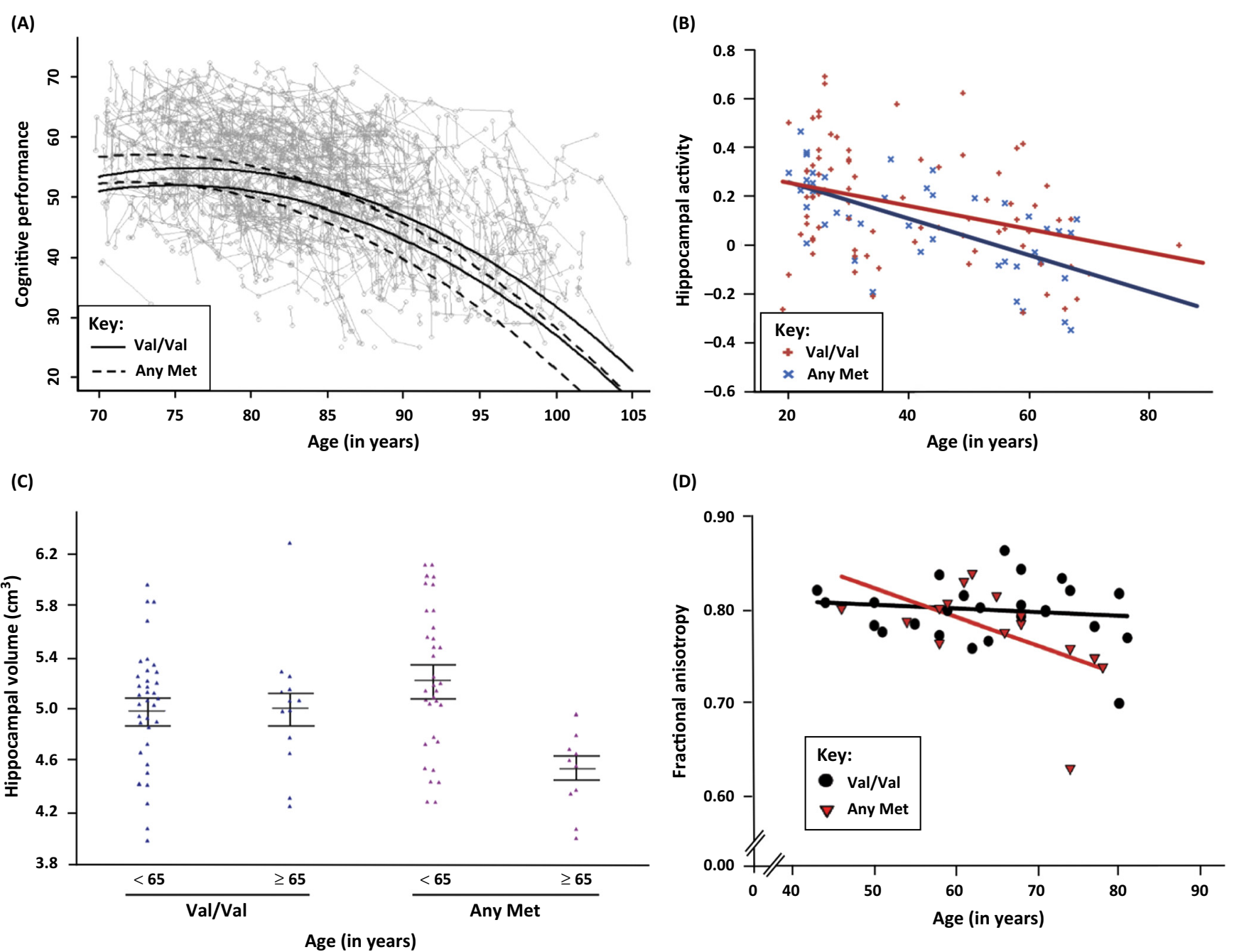

TRENDS in Cognitive Sciences

Figure 3. Effects of the brain-derived neurotrophic factor (BDNF) polymorphism on (A) longitudinal decline in perceptual speed across 13 years, with steeper decline for $B D N F$ Met carriers. Perceptual speed is measured using the digit-letter task, which requires participants to name letters associated with a digit, according to a template. The $y$-axis indicates the total number of correct responses after 3 minutes. Adapted from [37] with permission from American Psychological Association. Interaction between age and $B D N F$, reflecting (B) lower hippocampal activity during retrieval of episodic memories, (C) smaller hippocampal volumes, and (D) lower white-matter integrity in the splenium for older BDNF Met carriers. Hippocampal activity in (B) indicates parameter estimates of the blood-oxygen-level-dependent (BOLD) response measured in arbitrary units in left hippocampus, which is greater during retrieval relative to a baseline condition. White-matter integrity is indicated by fractional anisotropy. Adapted from [39] and [38] with permission from Nature Publishing Group, and from [43] with permission from Frontiers Research Foundation.

formation and consolidation [56]. In agreement with the resource-modulation hypothesis, a recent study reported that older adults carrying the KIBRA T allele showed better spatial learning compared with C-allele homozygotes, although no genotype effects were found in younger adults [57]. Another study also documented better episodic memory in older KIBRA T carriers than in C-allele homozygotes, but there was no effect of this polymorphism in a sample of older adults with mild cognitive impairment [58]. This pattern is consistent with the resource-modulation hypothesis and the prediction that genetic effects diminish once individuals approach dementia or death (Figure 1).

Larger effects of KIBRA on episodic memory in old age were replicated in a lifespan sample aged $35-85$ years, documenting an advantageous effect of the $\mathrm{T}$ allele on immediate free recall with advancing age [59]. In an fMRI subsample, T-allele carriers also exhibited increased hippocampal activity compared with $\mathrm{C}$ homozygotes during retrieval of episodic memories. However, KIBRA modulated episodic memory and hippocampal activation only in relatively younger elderly persons (aged 55-60 years). Despite age magnification of KIBRA effects on behavior in the larger sample, there was no genetic modulation of brain activity and memory in the scanner task in the older age group (aged 65-75 years). The authors speculated that older adults carrying the disadvantageous genotype may have increased hippocampal activation associated with pathological aging that overshadows genetic effects. This underscores the importance to screen for participants with dementia or prodromal dementia in this type of research.

Another lifespan study reported further evidence in favor of the resource-modulation hypothesis [60], both with respect to brain function and behavior. First, increasing age was associated with larger genetic effects on immediate and delayed free recall (Figure 4A,B). During an fMRI task, older $\mathrm{C}$ homozygotes had lower hippocampal activation during encoding and retrieval compared with younger 

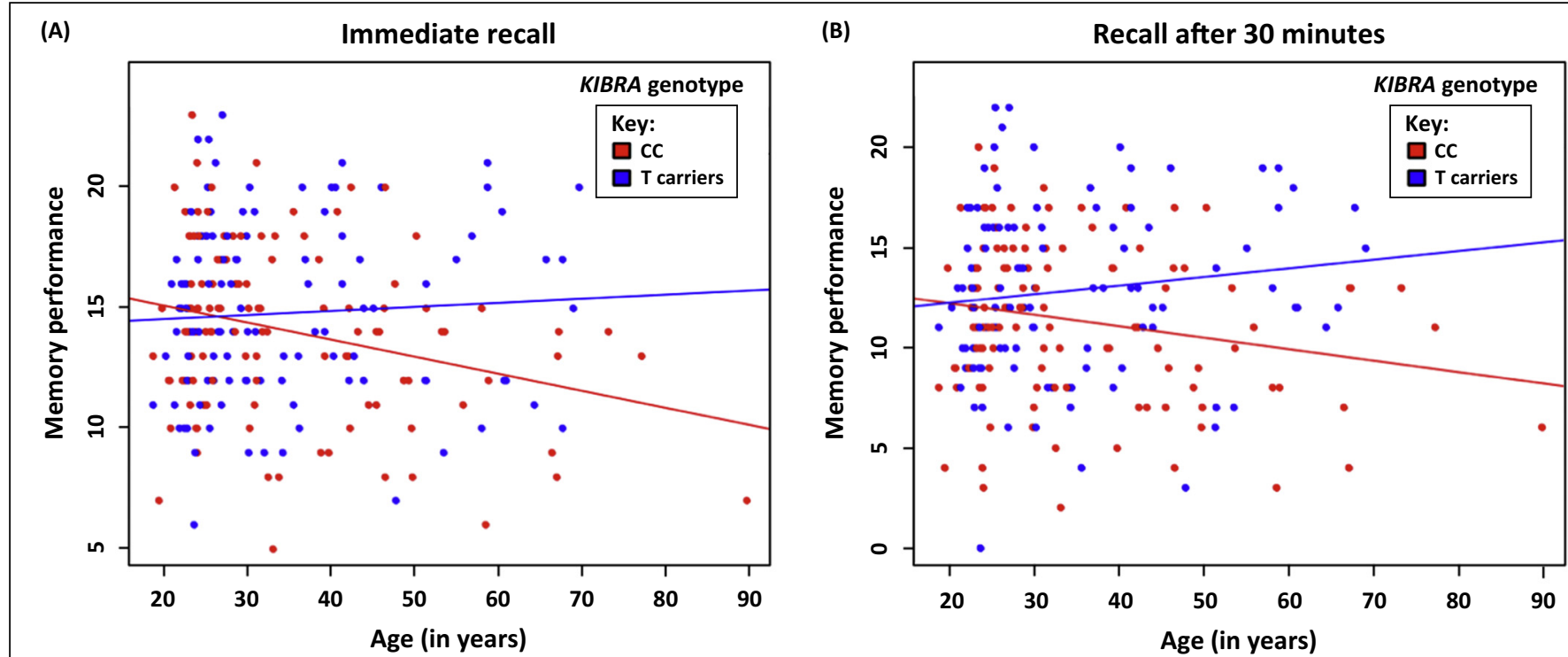

(c)

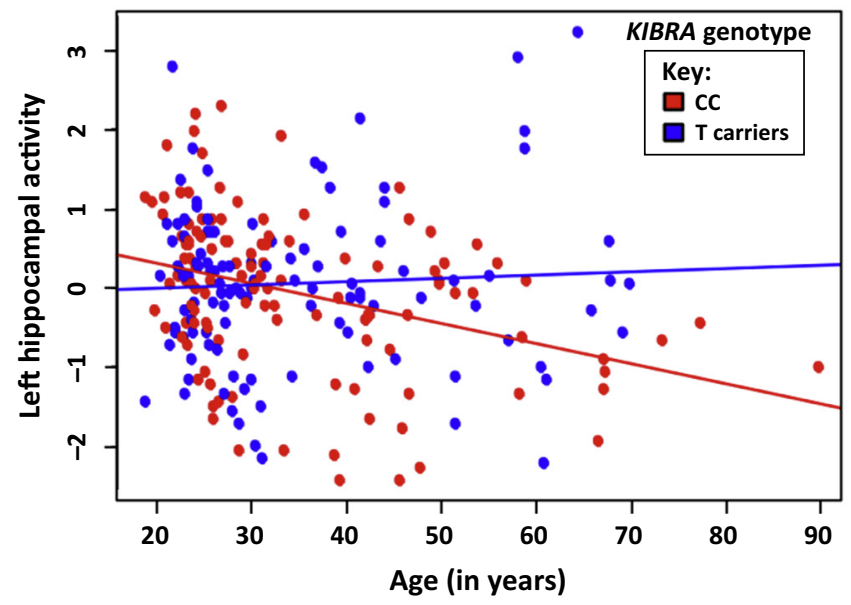

(D)

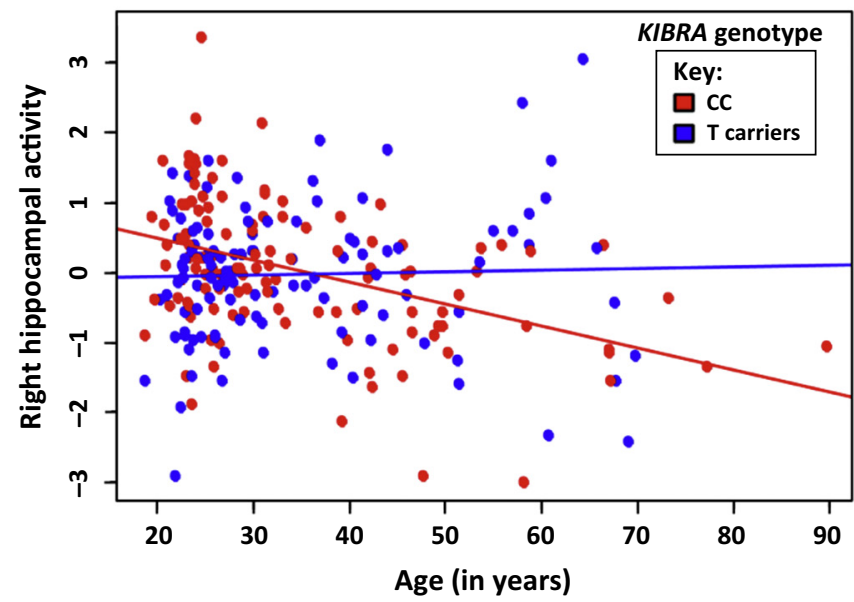

TRENDS in Cognitive Sciences

Figure 4. Groups with different kidney and brain expressed protein (KIBRA) genotypes show different correlations between increasing age and performance on (A) immediate and (B) 30 minute delayed recall of a story, as measured with the Wechsler Memory Scale. (C,D) KIBRA genotype group differences in the correlation between age and brain activation during an episodic memory task. (C) The KIBRA CC group (red) exhibits a negative correlation between age and activity in left hippocampus during encoding, which is not observed for T-allele carriers (blue). (D) The KIBRA CC group (red) exhibits a negative correlation between age and activity in right hippocampus during retrieval, which is not observed for T-allele carriers (blue). Hippocampal activity indicates parameter estimates of the BOLD response measured in arbitrary units, which is greater during encoding and retrieval relative to a baseline condition. Adapted from [60] with permission from Elsevier.

C homozygotes, demonstrating stronger genetic effects with advancing age (Figure 4C,D).

\section{Dopamine D2 receptor (DRD2) polymorphisms}

A behavioral study investigated the effect of genetic variation in the DRD2 gene on the ability to inhibit an action. Genetic predisposition for higher density of extrastriatal D2 receptors (DRD2 CC) was associated with better inhibition of unwanted action tendencies, an effect that was more pronounced in older than in younger adults (Figure 5) [61]. With respect to brain functioning, another variation in the DRD2 gene showed a similar pattern of age magnification, both on brain and behavior: lower performance in long-term memory updating was found for older carriers of the allele associated with fewer D2 receptors, as compared with non-carriers [62]. In addition, older risk carriers had lower brain activity in left caudate nucleus, a region crucial for updating [63]. Although there are relatively few studies investigating the effects of $D R D 2$, the available data suggest that this gene influences brain and cognition more strongly in older than in younger adults.

\section{Factors affecting age magnification of genetic effects}

As reviewed above, studies have often observed genetic effects in older, but not younger, adults. This may partly account for inconsistent findings in the extant literature because many studies have included younger participants only or collapsed the data across different age-cohorts. However, in age-comparative work, the available evidence is not unequivocal either, and a few studies have failed to find magnification of genetic effects on brain and cognition in aging (e.g., $[64,65])$. In the following we highlight factors that may limit or enhance the likelihood of observing magnified genetic effects in aging. 


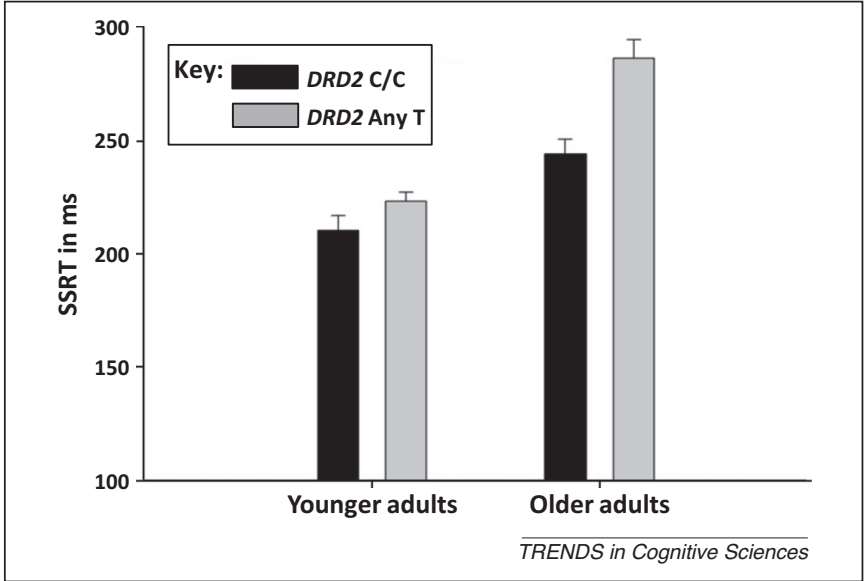

Figure 5. Age magnification of the effects of the dopamine receptor D2 (DRD2) polymorphism on inhibitory control (in $\mathrm{ms}$ ) measured by the stop-signal reaction time task (SSRT), with older T carriers (fewer dopamine D2 receptors) showing disproportionate slowing. Adapted from [61] with permission from Elsevier.

\section{Gene-gene interactions}

Most studies focus on the effects of single genes. However, genetic effects on cognition and brain may be particularly strong when carrying two or more disadvantageous genotypes. This could result in both additive and interactive effects, emphasizing the importance of investigating the effects of multiple genes.

Several studies have investigated the joint effects of DArelated genes on executive functioning, working memory, and episodic memory in old age [66-68]. For example, a DRD2 polymorphism interacted with a DA transporter gene in an episodic memory task requiring recall of words in backward order [68]: carriers of genetic predispositions for more D2 receptors and higher synaptic DA levels had higher recall accuracy. The main effects of each gene and the gene-gene interaction were larger in older than in younger adults. Because both the dopaminergic and glutamatergic systems modulate consolidation of episodic memories, one study investigated whether DRD2 and glutamate receptor genes interactively affect episodic memory [69]. A gene-gene interaction was observed in older adults only, with individuals carrying genotypes associated with greater DA and glutamate receptor efficacy showing the highest episodic memory performance. Adverse additive effects of $C O M T \mathrm{Val} / \mathrm{Val}, B D N F$ Met, and age have been reported for executive functioning, such that older adults with a high-risk combination performed particularly poorly. These effects were strengthened by the presence of the $A P O E$ e4 allele [70]. Regarding neuroimaging data, studies investigating additive or interactive genetic effects in old age are rare. However, data suggest that considering more than one gene may help to explain variance in brain activity: hippocampal activity during episodic encoding decreased as a function of the number of $A P O E$ e4 and $B D N F$ Met alleles (none, one, or both), yielding stronger effects than those of the individual genes [71].

\section{Study population characteristics}

Another factor that may contribute to inconsistent findings across studies concerns participant characteristics. Although some studies include population-based samples or even individuals with different diseases, other studies screen their subjects carefully, which may result in greater participant selectivity. Crucially, most genetic studies on brain and cognition did not control for incident dementia. This is a serious omission, given that the prodromal phase of dementia might start several years, if not decades, before a clinical diagnosis is rendered [72]. As predicted by the resource-modulation model, genetic effects may diminish once individuals reach very low resource levels, as in dementia or terminal decline (Figure 1).

In general, genetic effects may be easier to detect when stratifying individuals according to different characteristics. For instance, interactive effects between two memoryrelated genes, KIBRA and calsyntenin 2 (CLSTN2), were observed for episodic memory in older adults having relatively mild depression, with individuals carrying both risk alleles performing the worst [73]. By contrast, no genetic effects were observed in non-depressed individuals, suggesting that such effects are most easily detected at suboptimal levels of brain integrity, in this case among older persons with mild depression. Relatedly, imaging studies report stronger effects of COMT in populations with reduced brain resources [74-76] compared with healthy controls: in patients with major depressive disorder [76] and panic disorder [75], white-matter integrity was lower for Val homozygotes than for Met carriers, whereas no $C O M T$ effects were observed for healthy controls. Thus, different study population characteristics may affect whether or not a genetic effect is observed.

\section{Gene-environment interactions}

Detrimental effects of disadvantageous genotypes can be counteracted through an advantageous lifestyle. For instance, physical activity has been shown to attenuate negative effects of different genes on both episodic memory [77] and working memory [78]. Similarly, higher levels of education and lifetime intellectual enrichment counteracted the effects of $A P O E$ e4 on dementia occurrence [79] and cognitive decline [80]. Furthermore, higher blood pressure and increased cardiovascular risk have been shown to interact with risk alleles of $A P O E$ [81,82], $B D N F$ [83], and KIBRA [84] in affecting cognition negatively. A healthier diet has also been reported to enhance the protective effects linked to carrying $A P O E$ e 2 or e3 alleles with regard to cognitive performance [85]. Imaging studies investigating interactive effects between genes and environmental factors in older age are scarce. However, one study reported that older APOE e4 carriers who are more physically active had higher activity in different taskrelated brain regions during an episodic memory task than non-carriers or those with lower physical activity levels. In line with this pattern, structural imaging data demonstrate that increased physical activity is protective against the detrimental effects of $A P O E$ e 4 on hippocampal volume across 18 months [86]. Another study showed that higher midlife cognitive activity attenuated amyloid accumulation in e4 carriers compared with non-carriers [87].

\section{Epigenetics}

By addressing epigenetic mechanisms [88], research may contribute to our understanding of how environmental and 
lifestyle factors affect gene expression, and this may influence whether or not age magnification of genetic effects is observed. Interestingly, twin studies show that monozygotic twins become more discordant in aging with respect to their DNA methylation profiles, suggesting that different lifestyles may regulate gene expression across the lifespan [89]. Note, however, that gene-environment interactions might occur without any epigenetic modification. Moreover, individual differences in epigenetic mechanisms may also be inherited [90]. Therefore, it is vital to show longitudinally that lifestyle changes or interventions directly affect expression of a particular gene through epigenetic mechanisms, and consequently brain and cognition. Given that experimental control of lifestyle factors and their effects on epigenetic mechanisms is extremely difficult to achieve in humans, most findings on epigenetic mechanisms are based on animal data $[88,91]$.

\section{Gene-environment correlations}

Gene-environment correlations indicate that a particular genotype is more frequently associated with a particular environment. For instance, individuals with more advantageous genetic predispositions may actively seek a more stimulating environment. Environmental exposure may, in turn, enhance expression of a particular gene via epigenetic mechanisms, thereby increasing inter-individual differences [92]. Such gene-environment interactions may partly account for the increased heritability of cognitive measures in old age, as supported by simulation work [93] and a meta-analysis of correlational ratios in twin studies [6]. Although genetically identical twins may choose similar environments resulting in relatively stable monozygotic (MZ) correlations, dizygotic (DZ) twins may become more different over time owing to different genetically driven environmental choices, leading to a lower correlation between dizygotic twins. Thus, over time, the correlation ratios (MZ/DZ) may increase, suggesting increased heritability in late adulthood, although environmental factors contribute to the enhanced association.

\section{Concluding remarks}

Increasing evidence suggests that effects of common genetic variations on brain and behavior become stronger in late life, supporting the resource-modulation hypothesis.

\section{Box 1. Outstanding questions}

- Do genetic effects diminish once individuals approach cognitive decline and death? This particular prediction of the resource-modulation hypothesis has not been extensively tested.

- Given that genes may affect both brain structure and function, multimodal imaging studies are necessary to understand the temporal dynamics of genetic effects on brain functioning. Does a particular gene affect brain structure and function independently, or are the functional effects mediated through effects on brain structure?

- Given that genetic variations may differ across generations, longitudinal studies are necessary to replicate the patterns of age magnification observed in cross-sectional studies.

- Is there a direct relationship between mean changes in brain resources and heritability estimates of cognition? Do lower brain resources per se lead to increased genetic effects in old age, or are these mediated through lifestyle and epigenetic factors?
Similar patterns have been reported in other populations characterized by reduced brain resources, by contrasting samples with different diseases to healthy controls. So far, the bulk of relevant studies are cross-sectional. Longitudinal behavioral, structural, and functional imaging studies will be necessary to confirm the patterns reported in the cross-sectional data (Box 1). Furthermore, some of the inconsistent patterns reported in candidate gene studies likely stem from gene-gene interactions, and from environmental and lifestyle factors resulting in epigenetic differences. Behavioral and multimodal brain imaging research across long follow-up intervals that target the operation of these factors during the transition from early to late adulthood constitutes a key avenue for future research.

\section{Acknowledgments}

Preparation of this review was supported by grants from the Swedish Research Council, the Swedish Research Council for Health, Working Life, and Welfare, Swedish Brain Power, an Alexander von Humboldt Research Award, and a donation from the af Jochnick Foundation to L.B., and from grants from the Federal Ministry of Education and Research and a Gottfried Wilhelm Leibniz Award 2010 of the German Research Foundation (DFG) to U.L.

\section{References}

1 Rönnlund, M. et al. (2005) Stability, growth, and decline in adult life span development of declarative memory: cross-sectional and longitudinal data from a population-based study. Psychol. Aging 20, $3-18$

2 Lindenberger, U. et al. (2013) Heterogeneity in frontal lobe aging. In Principles of Frontal Lobe Functions (Stuss, D.T. and Knight, R.T., eds), pp. 609-627, Oxford University Press

3 Fandakova, Y. et al. (2014) Maintenance of youth-like processing protects against false memory in later adulthood. Neurobiol. Aging 36, 933-941

4 Nyberg, L. et al. (2012) Memory aging and brain maintenance. Trends Cogn. Sci. 16, 292-305

5 Finkel, D. et al. (1998) Longitudinal and cross-sectional twin data on cognitive abilities in adulthood: the Swedish Adoption/Twin Study of Aging. Dev. Psychol. 34, 1400-1413

6 Reynolds, C.A. and Finkel, D. (2015) A meta-analysis of heritability of cognitive aging: minding the 'missing heritability' gap. Neuropsychol. Rev. 25, 97-112

7 McClearn, G.E. et al. (1997) Substantial genetic influence on cognitive abilities in twins 80 or more years old. Science $276,1560-1563$

8 Tucker-Drob, E.M. et al. (2014) Shared and unique genetic and environmental influences on aging-related changes in multiple cognitive abilities. Dev. Psychol. 50, 152-166

9 Batouli, S.A. et al. (2014) The heritability of volumes of brain structures and its relationship to age: a review of twin and family studies. Ageing Res. Rev. 13, 1-9

10 Kremen, W.S. et al. (2012) Heritability of brain ventricle volume: converging evidence from inconsistent results. Neurobiol. Aging 33, 1-8

11 Vogler, C. et al. (2014) Substantial SNP-based heritability estimates for working memory performance. Transl. Psychiatry 4, e438

12 Davies, G. et al. (2011) Genome-wide association studies establish that human intelligence is highly heritable and polygenic. Mol. Psychiatry $16,996-1005$

13 Wilson, R.S. et al. (2011) Heritability of different forms of memory in the Late Onset Alzheimer's Disease Family Study. J. Alzheimers Dis. 23, 249-255

14 Lindenberger, U. et al. (2008) Age-related decline in brain resources modulates genetic effects on cognitive functioning. Front. Neurosci. 2 , 234-244

15 Rasch, B. et al. (2010) Imaging genetics of cognitive functions: focus on episodic memory. Neuroimage 53, 870-877

$16 \mathrm{Bu}, \mathrm{G}$. (2009) Apolipoprotein $\mathrm{E}$ and its receptors in Alzheimer's disease: pathways, pathogenesis and therapy. Nat. Rev. Neurosci. 10, 333-344 
17 Okuizumi, K. et al. (1995) Genetic association of the very low density lipoprotein (VLDL) receptor gene with sporadic Alzheimer's disease. Nat. Genet. 11, 207-209

18 Blacker, D. et al. (1997) ApoE-4 and age at onset of Alzheimer's disease: the NIMH genetics initiative. Neurology 48, 139-147

19 Dik, M.G. et al. (2000) Stroke and apolipoprotein E epsilon4 are independent risk factors for cognitive decline: a population-based study. Stroke 31, 2431-2436

20 Bretsky, P. et al. (2003) The role of APOE-epsilon4 in longitudinal cognitive decline: MacArthur Studies of Successful Aging. Neurology 60, 1077-1081

21 Wisdom, N.M. et al. (2011) The effects of apolipoprotein E on nonimpaired cognitive functioning: a meta-analysis. Neurobiol. Aging 32, 63-74

22 Liu, F. et al. (2010) The apolipoprotein E gene and its age-specific effects on cognitive function. Neurobiol. Aging 31, 1831-1833

23 Schiepers, O.J. et al. (2012) APOE E4 status predicts age-related cognitive decline in the ninth decade: longitudinal follow-up of the Lothian Birth Cohort 1921. Mol. Psychiatry 17, 315-324

24 Zhang, C. and Pierce, B.L. (2014) Genetic susceptibility to accelerated cognitive decline in the US Health and Retirement Study. Neurobiol. Aging 35, 1512-1518

25 De Jager, P.L. et al. (2012) A genome-wide scan for common variants affecting the rate of age-related cognitive decline. Neurobiol. Aging 33, e1011-e1015

26 Filippini, N. et al. (2011) Differential effects of the APOE genotype on brain function across the lifespan. Neuroimage 54, 602-610

27 Nichols, L.M. et al. (2012) Interactive effect of apolipoprotein e genotype and age on hippocampal activation during memory processing in healthy adults. Arch. Gen. Psychiatry 69, 804-813

28 Grady, C. (2012) The cognitive neuroscience of ageing. Nat. Rev. Neurosci. 13, 491-505

29 Crivello, F. et al. (2010) Effects of ApoE-epsilon4 allele load and age on the rates of grey matter and hippocampal volumes loss in a longitudinal cohort of 1186 healthy elderly persons. Neuroimage 53, 1064-1069

30 Cohen, R.M. et al. (2001) Effect of apolipoprotein E genotype on hippocampal volume loss in aging healthy women. Neurology 57, 2223-2228

31 MacDonald, S.W. et al. (2011) Trajectories of cognitive decline following dementia onset: what accounts for variation in progression? Dement. Geriatr. Cogn. Disord. 31, 202-209

32 Bunce, D. et al. (2004) APOE and cognitive decline in preclinical Alzheimer disease and non-demented aging. Neurology 63, $816-821$

33 Tyler, W.J. et al. (2002) From acquisition to consolidation: on the role of brain-derived neurotrophic factor signaling in hippocampal-dependent learning. Learn. Mem. 9, 224-237

34 Egan, M.F. et al. (2003) The BDNF val66met polymorphism affects activity-dependent secretion of BDNF and human memory and hippocampal function. Cell 112, 257-269

35 Kambeitz, J.P. et al. (2012) Effect of BDNF val(66)met polymorphism on declarative memory and its neural substrate: a meta-analysis. Neurosci. Biobehav. Rev. 36, 2165-2177

$36 \mathrm{Li}$, S-C. et al. (2010) Ebbinghaus revisited: influences of the BDNF Val66Met polymorphism on backward serial recall are modulated by human aging. J. Cogn. Neurosci. 22, 2164-2173

37 Ghisletta, P. et al. (2014) The Val/Met polymorphism of the brainderived neurotrophic factor (BDNF) gene predicts decline in perceptual speed in older adults. Psychol. Aging 29, 384-392

38 Sanchez, M.M. et al. (2011) BDNF polymorphism predicts the rate of decline in skilled task performance and hippocampal volume in healthy individuals. Transl. Psychiatry 1, e51

39 Sambataro, F. et al. (2010) BDNF modulates normal human hippocampal ageing. Mol. Psychiatry 15, 116-118

40 Ikeda, O. et al. (2002) Effects of brain-derived neurotrophic factor (BDNF) on compression-induced spinal cord injury: BDNF attenuates down-regulation of superoxide dismutase expression and promotes up-regulation of myelin basic protein expression. J. Neuropathol. Exp. Neurol. 61, 142-153

41 Morse, J.K. et al. (1993) Brain-derived neurotrophic factor (BDNF) prevents the degeneration of medial septal cholinergic neurons following fimbria transection. J. Neurosci. 13, 4146-4156
42 Lim, Y.Y.et al. (2014) Effect of BDNF Val66Met on memory decline and hippocampal atrophy in prodromal Alzheimer's disease: a preliminary study. PLoS ONE 9, e86498

43 Kennedy, K.M. et al. (2009) BDNF Val66Met polymorphism influences age differences in microstructure of the corpus callosum. Front. Hum. Neurosci. 3, 19

44 Matsumoto, M. et al. (2003) Catechol O-methyltransferase mRNA expression in human and rat brain: evidence for a role in cortical neuronal function. Neuroscience 116, 127-137

45 Tunbridge, E.M. et al. (2006) Catechol-O-methyltransferase, cognition, and psychosis: Val158Met and beyond. Biol. Psychiatry 60, 141-151

46 Egan, M.F. et al. (2001) Effect of COMT Val108/158 Met genotype on frontal lobe function and risk for schizophrenia. Proc. Natl. Acad. Sci. U.S.A. 98, 6917-6922

47 Lotta, T. et al. (1995) Kinetics of human soluble and membrane-bound catechol O-methyltransferase: a revised mechanism and description of the thermolabile variant of the enzyme. Biochemistry 34, 4202-4210

48 Nagel, I.E. et al. (2008) Human aging magnifies genetic effects on executive functioning and working memory. Front. Hum. Neurosci. 2, 1

49 Störmer, V.S. et al. (2011) Dopaminergic and cholinergic modulations of visual-spatial attention and working memory: insights from molecular genetic research and implications for adult cognitive development. Dev. Psychol. 48, 875-889

50 Papenberg, G. et al. (2013) COMT polymorphism and memory dedifferentiation in old age. Psychol. Aging 29, 347-383

51 de Frias, C.M. et al. (2005) Catechol O-methyltransferase Val158Met polymorphism is associated with cognitive performance in nondemented adults. J. Cogn. Neurosci. 17, 1018-1025

52 Josefsson, M. et al. (2012) Genetic and lifestyle predictors of 15-year longitudinal change in episodic memory. J. Am. Geriatr. Soc. 60, 2308-2312

53 Sambataro, F. et al. (2009) Catechol-O-methyltransferase valine(158)methionine polymorphism modulates brain networks underlying working memory across adulthood. Biol. Psychiatry 66, $540-548$

54 Papenberg, G. et al. (2014) Magnified effects of the COMT gene on white-matter microstructure in very old age. Brain Struct. Funct.

55 Papassotiropoulos, A. et al. (2006) Common Kibra alleles are associated with human memory performance. Science $314,475-478$

56 Schneider, A. et al. (2010) KIBRA: a new gateway to learning and memory? Front. Aging Neurosci. 2, 4

57 Schuck, N.W. et al. (2013) Aging and KIBRA/WWC1 genotype affect spatial memory processes in a virtual navigation task. Hippocampus 23, 919-930

58 Almeida, O.P. et al. (2008) KIBRA genetic polymorphism influences episodic memory in later life, but does not increase the risk of mild cognitive impairment. J. Cell. Mol. Med. 12, 1672-1676

59 Kauppi, K. et al. (2011) KIBRA polymorphism is related to enhanced memory and elevated hippocampal processing. J. Neurosci. 31, 1421814222

60 Muse, J. et al. (2014) WWC1 genotype modulates age-related decline in episodic memory function across the adult life span. Biol. Psychiatry $75,693-700$

61 Colzato, L.S. et al. (2013) The genetic impact (C957T-DRD2) on inhibitory control is magnified by aging. Neuropsychologia 51, $1377-1381$

62 Persson, J. et al. (2014) Influences of a DRD2 polymorphism on updating of long-term memory representations and caudate BOLD activity: magnification in aging. Hum. Brain Mapp. 36, 1325-1334

63 Bäckman, L. et al. (2011) Effects of working-memory training on striatal dopamine release. Science 333, 718

64 Laukka, E.J. et al. (2013) Genetic effects on old-age cognitive functioning: a population-based study. Psychol. Aging 28, 262-274

65 Das, D. et al. (2014) Cognitive ability, intraindividual variability, and common genetic variants of catechol-O-methyltransferase and brainderived neurotrophic factor: a longitudinal study in a population-based sample of older adults. Psychol. Aging 29, 393-403

66 Papenberg, G. et al. (2013) Dopaminergic gene polymorphisms affect long-term forgetting in old age: further support for the magnification hypothesis. J. Cogn. Neurosci. 25, 571-579

67 Greenwood, P.M. et al. (2014) Healthy aging increases the cognitive effects of two genes that influence extracellular dopamine. Psychol. Aging 29, 363-373 
$68 \mathrm{Li}$, S-C. et al. (2012) Aging magnifies the effects of dopamine transporter and D2 receptor genes on backward serial memory. Neurobiol. Aging 34, e1-e10

69 Papenberg, G. et al. (2014) Dopamine and glutamate receptor genes interactively influence episodic memory in old age. Neurobiol. Aging 35, e1213-e1218

70 Sapkota, S. et al. (2015) Synergistic associations of catechol-Omethyltransferase and brain-derived neurotrophic factor with executive function in aging are selective and modified by apolipoprotein E. Neurobiol. Aging 36, 249-256

71 Kauppi, K. et al. (2014) Additive genetic effect of APOE and BDNF on hippocampus activity. Neuroimage 89, 306-313

72 Thorvaldsson, V. et al. (2011) Onset and rate of cognitive change before dementia diagnosis: findings from two Swedish population-based longitudinal studies. J. Int. Neuropsychol. Soc. 17, 154-162

73 Pantzar, A et al. (2014) Interactive effects of KIBRA and CLSTN2 polymorphisms on episodic memory in old-age unipolar depression. Neuropsychologia 62, 137-142

74 Ceaser, A. et al. (2013) COMT influences on prefrontal and striatal blood oxygenation level-dependent responses during working memory among individuals with schizophrenia, their siblings, and healthy controls. Cogn. Neuropsychiatry 18, 257-283

$75 \mathrm{Kim}, \mathrm{B}$. et al. (2013) The effects of the catechol-O-methyltransferase val158met polymorphism on white matter connectivity in patients with panic disorder. J. Affect. Disord. 147, 64-71

76 Seok, J.H. et al. (2013) Effect of the COMT val158met polymorphism on white matter connectivity in patients with major depressive disorder Neurosci. Lett. 545, 35-39

77 Ferencz, B. et al. (2014) The benefits of staying active in old age: physical activity counteracts the negative influence of PICALM, BIN1, and CLU risk alleles on episodic memory functioning. Psychol. Aging 29, 440-449

78 Erickson, K.I. et al. (2013) The brain-derived neurotrophic factor Val66Met polymorphism moderates an effect of physical activity on working memory performance. Psychol. Sci. 24, 1770-1779

79 Wang, H.X. et al. (2012) Education halves the risk of dementia due to apolipoprotein epsilon4 allele: a collaborative study from the Swedish brain power initiative. Neurobiol. Aging 33, e1001-e1007
80 Vemuri, P. et al. (2014) Association of lifetime intellectual enrichment with cognitive decline in the older population. JAMA Neurol. 71, 10171024

81 de Frias, C.M. et al. (2014) Hypertension moderates the effect of APOE on 21-year cognitive trajectories. Psychol. Aging 29, 431-439

$82 \mathrm{McF}$ all, G.P. et al. (2014) $\mathrm{ApoE}$ and pulse pressure interactively influence level and change in the aging of episodic memory: protective effects among epsilon2 carriers. Neuropsychology 29, 388-401

$83 \mathrm{Raz}$, N. et al. (2009) Genetic and vascular modifiers of age-sensitive cognitive skills: Effects of COMT, BDNF, ApoE, and hypertension. Neuropsychology 23, 105-116

84 Wersching, H. et al. (2011) Impact of common KIBRA allele on human cognitive functions. Neuropsychopharmacology 36, 1296-1304

85 Whalley, L.J. et al. (2008) n-3 Fatty acid erythrocyte membrane content, APOE varepsilon4, and cognitive variation: an observational follow-up study in late adulthood. Am. J. Clin. Nutr. $87,449-454$

86 Smith, J.C. et al. (2014) Physical activity reduces hippocampal atrophy in elders at genetic risk for Alzheimer's disease. Front. Aging Neurosci. 6, 61

87 Wirth, M. et al. (2014) Gene-environment interactions: lifetime cognitive activity, APOE genotype, and beta-amyloid burden. J. Neurosci. 34, 8612-8617

88 Sweatt, J.D. (2013) The emerging field of neuroepigenetics. Neuron 80 , 624-632

89 Talens, R.P. et al. (2012) Epigenetic variation during the adult lifespan: cross-sectional and longitudinal data on monozygotic twin pairs. Aging Cell 11, 694-703

90 Bell, J.T. and Spector, T.D. (2012) DNA methylation studies using twins: what are they telling us? Genome Biol. 13, 172

91 Spiegel, A.M. et al. (2014) Epigenetic contributions to cognitive aging: disentangling mindspan and lifespan. Learn. Mem. 21, 569-574

92 Deater-Deckard, K. and Mayr, U. (2005) Cognitive change in aging: identifying gene-environment correlation and nonshared environment mechanisms. J. Gerontol. B: Psychol. Sci. Soc. Sci. 60, 24-31

93 Beam, C.R. and Turkheimer, E. (2013) Phenotype-environment correlations in longitudinal twin models. Dev. Psychopathol. 25, 7-16 